PROCEEDINGS OF THE

AMERICAN MATHEMATICAL SOCIETY

Volume 138, Number 7, July 2010, Pages 2265-2268

S 0002-9939(10)10323-2

Article electronically published on February 23, 2010

\title{
ON THE EXISTENCE OF EMBEDDINGS INTO MODULES OF FINITE HOMOLOGICAL DIMENSIONS
}

\author{
RYO TAKAHASHI, SIAMAK YASSEMI, AND YUJI YOSHINO
}

(Communicated by Bernd Ulrich)

\begin{abstract}
Let $R$ be a commutative Noetherian local ring. We show that $R$ is Gorenstein if and only if every finitely generated $R$-module can be embedded in a finitely generated $R$-module of finite projective dimension. This extends a result of Auslander and Bridger to rings of higher Krull dimension, and it also improves a result due to Foxby where the ring is assumed to be CohenMacaulay.
\end{abstract}

\section{INTRODUCTION}

Throughout this paper, let $R$ be a commutative Noetherian local ring. All $R$-modules in this paper are assumed to be finitely generated.

In [1, Proposition 2.6 (a) and (d)] Auslander and Bridger proved the following.

Theorem 1.1 (Auslander-Bridger). The following are equivalent:

(1) $R$ is quasi-Frobenius (i.e. Gorenstein with Krull dimension zero).

(2) Every $R$-module can be embedded in a free R-module.

On the other hand, in [4, Theorem 2] Foxby showed the following.

Theorem 1.2 (Foxby). The following are equivalent:

(1) $R$ is Gorenstein.

(2) $R$ is Cohen-Macaulay, and every $R$-module can be embedded in an $R$-module of finite projective dimension.

For an $R$-module $C$ we denote by $\operatorname{add}_{R} C$ the class of $R$-modules which are direct summands of finite direct sums of copies of $C$. The $C$-dimension of an $R$-module $X, C-\operatorname{dim}_{R} X$, is defined as the infimum of nonnegative integers $n$ such that there exists an exact sequence

$$
0 \rightarrow C_{n} \rightarrow C_{n-1} \rightarrow \cdots \rightarrow C_{0} \rightarrow X \rightarrow 0
$$

of $R$-modules with $C_{i} \in \operatorname{add}_{R} C$ for all $0 \leq i \leq n$.

In this paper, we prove the following theorem. This result removes from Theorem 1.2 the assumption that $R$ is Cohen-Macaulay, and it extends Theorem 1.1 to

Received by the editors November 26, 2008, and, in revised form, May 24, 2009.

2010 Mathematics Subject Classification. Primary 13D05, 13H10.

Key words and phrases. Gorenstein ring, Cohen-Macaulay ring, projective dimension, injective dimension, (semi)dualizing module.

The first and second authors were supported in part by Grant-in-Aid for Young Scientists (B) 19740008 from JSPS and by grant No. 88013211 from IPM, respectively.

(C)2010 American Mathematical Society Reverts to public domain 28 years from publication 
rings of higher Krull dimension. It should be noted that our proof of this result is different from Foxby's proof for the special case $C=R$.

Theorem 1.3. Let $R$ be a commutative Noetherian local ring with residue field $k$. Let $C$ be a semidualizing $R$-module of depth $t$. Then the following are equivalent:

(1) $C$ is dualizing.

(2) Every $R$-module can be embedded in an $R$-module of finite $C$-dimension.

(3) The $R$-module $\operatorname{Tr} \Omega^{t} k \otimes_{R} C$ can be embedded in an $R$-module of finite $C$ dimension. (Here $\operatorname{Tr} \Omega^{t} k$ denotes the transpose of the $t$-th syzygy of the $R$-module k.)

Moreover, if one of these three conditions holds, then $R$ is Cohen-Macaulay.

\section{Proof of Theorem 1.3 and its applications}

First of all, we recall the definition of a semidualizing module.

Definition 2.1. An $R$-module $C$ is called semidualizing if the natural homomorphism $R \rightarrow \operatorname{Hom}_{R}(C, C)$ is an isormophism and $\operatorname{Ext}_{R}^{i}(C, C)=0$ for all $i>0$.

Note that a dualizing module is nothing but a semidualizing module of finite injective dimension. Another typical example of a semidualizing module is a free module of rank one. Recently a considerable number of authors have studied semidualizing modules and have obtained many results concerning these modules.

We denote by $\mathfrak{m}$ the maximal ideal of $R$ and by $k$ the residue field of $R$. To prove our main theorem, we establish two lemmas.

Lemma 2.2. Let $C$ be a semidualizing $R$-module. Let $g: M \rightarrow X$ be an injective homomorphism of $R$-modules with $C$ - $\operatorname{dim}_{R} X<\infty$. If $\operatorname{Ext}_{R}^{i}(M, C)=0$ for any $1 \leq i \leq C-\operatorname{dim}_{R} X$, then the natural map $\lambda_{M}: M \rightarrow \operatorname{Hom}_{R}\left(\operatorname{Hom}_{R}(M, C), C\right)$ is injective.

Proof. First of all we prove that $M$ can be embedded in a module $C_{0}$ in $\operatorname{add}_{R} C$. For this we set $n=C-\operatorname{dim}_{R} X$. If $n=0$, then this is obvious from the assumption, since $X \in \operatorname{add}_{R} C$. If $n>0$, then there exists an exact sequence

$$
0 \rightarrow C_{n} \stackrel{d_{n}}{\rightarrow} C_{n-1} \stackrel{d_{n-1}}{\rightarrow} \cdots \stackrel{d_{1}}{\rightarrow} C_{0} \stackrel{d_{0}}{\rightarrow} X \rightarrow 0
$$

with $C_{i} \in \operatorname{add}_{R} C$ for $0 \leq i \leq n$. Putting $X_{i}=\operatorname{Im} d_{i}$, we have exact sequences

$$
0 \rightarrow X_{i+1} \rightarrow C_{i} \rightarrow X_{i} \rightarrow 0 \quad(0 \leq i \leq n-1) .
$$

Then we have $\operatorname{Ext}_{R}^{1}\left(M, X_{1}\right)=0$, since there are isomorphisms $\operatorname{Ext}_{R}^{1}\left(M, X_{1}\right) \cong$ $\operatorname{Ext}_{R}^{2}\left(M, X_{2}\right) \cong \ldots \cong \operatorname{Ext}_{R}^{n}\left(M, X_{n}\right) \cong \operatorname{Ext}_{R}^{n}\left(M, C_{n}\right)=0$. Hence $\operatorname{Hom}_{R}\left(M, d_{0}\right)$ : $\operatorname{Hom}_{R}\left(M, C_{0}\right) \rightarrow \operatorname{Hom}_{R}(M, X)$ is surjective. This implies that the homomorphism $g \in \operatorname{Hom}_{R}(M, X)$ is lifted to $f \in \operatorname{Hom}_{R}\left(M, C_{0}\right)$, i.e. $d_{0} \cdot f=g$. Since $g$ is injective, $f$ is injective as well. Therefore $M$ has an embedding $f$ into $C_{0}$.

To prove that $\lambda_{M}$ is injective, we note that $\lambda_{C_{0}}$ is an isomorphism because of $C_{0} \in \operatorname{add}_{R} C$. Since there is an injective homomorphism $f: M \rightarrow C_{0}$, the following commutative diagram forces $\lambda_{M}$ to be injective:

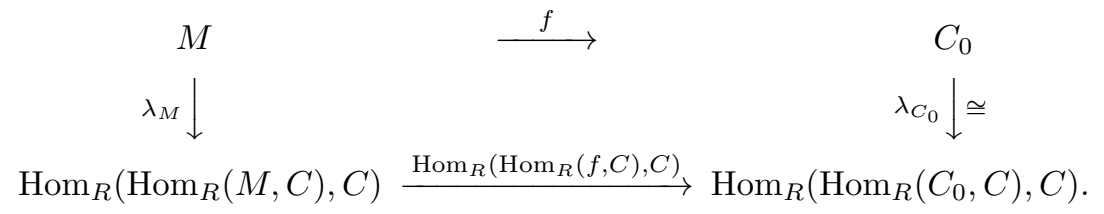


Lemma 2.3. Let $C$ be a semidualizing $R$-module and let $M$ be an $R$-module. Assume that $M$ is free on the punctured spectrum of $R$. Then there is an isomorphism

$$
\operatorname{Ext}_{R}^{i}(M, R) \cong \operatorname{Ext}_{R}^{i}\left(M \otimes_{R} C, C\right)
$$

for each integer $i \leq \operatorname{depth}_{R} C$.

Proof. Set $t=\operatorname{depth}_{R} C$. Since $C$ is semidualizing, we have a spectral sequence

$$
E_{2}^{p, q}=\operatorname{Ext}_{R}^{p}\left(\operatorname{Tor}_{q}^{R}(M, C), C\right) \Rightarrow \operatorname{Ext}_{R}^{p+q}(M, R) .
$$

Note by assumption that the $R$-module $\operatorname{Tor}_{q}^{R}(M, C)$ has finite length for $q>0$. By 2. Proposition 1.2.10(e)] we have $E_{2}^{p, q}=0$ if $p<t$ and $q>0$. Hence

$$
\operatorname{Ext}_{R}^{i}\left(M \otimes_{R} C, C\right)=E_{2}^{i, 0} \cong \operatorname{Ext}_{R}^{i}(M, R)
$$

for $i \leq t$.

Let $M$ be an $R$-module. Take a free resolution

$$
F_{\bullet}=\left(\cdots \stackrel{d_{n+1}}{\rightarrow} F_{n} \stackrel{d_{n}}{\rightarrow} F_{n-1} \stackrel{d_{n-1}}{\rightarrow} \cdots \stackrel{d_{1}}{\rightarrow} F_{0} \rightarrow 0\right)
$$

of $M$. Then for a nonnegative integer $n$ we define the $n$-th syzygy of $M$ by the image of $d_{n}$ and denote it by $\Omega_{R}^{n} M$ or simply by $\Omega^{n} M$. We also define the (Auslander) transpose of $M$ by the cokernel of the map $\operatorname{Hom}_{R}\left(d_{1}, R\right): \operatorname{Hom}_{R}\left(F_{0}, R\right) \rightarrow$ $\operatorname{Hom}_{R}\left(F_{1}, R\right)$ and denote it by $\operatorname{Tr}_{R} M$ or simply by $\operatorname{Tr} M$. Note that the $n$th syzygy and the transpose of $M$ are uniquely determined up to free summand. Note also that they commute with localization; namely, for every prime ideal $\mathfrak{p}$ of $R$ there are isomorphisms $\left(\Omega_{R}^{n} M\right)_{\mathfrak{p}} \cong \Omega_{R_{\mathfrak{p}}}^{n} M_{\mathfrak{p}}$ and $\left(\operatorname{Tr}_{R} M\right)_{\mathfrak{p}} \cong \operatorname{Tr}_{R_{\mathfrak{p}}} M_{\mathfrak{p}}$ up to free summand.

Recall that for a positive integer $n$ an $R$-module is called $n$-torsionfree if

$$
\operatorname{Ext}_{R}^{i}(M, R)=0
$$

for all $1 \leq i \leq n$. Now we can prove our main theorem.

Proof of Theorem [1.3. (1) $\Rightarrow(2)$ : By virtue of [6, Theorem (3.11)], the local ring $R$ is Cohen-Macaulay. Now assertion (2) follows from [4, Theorem 1].

$(2) \Rightarrow(3)$ : This implication is obvious.

$(3) \Rightarrow(1)$ : We denote by $(-)^{\dagger}$ the $C$-dual functor $\operatorname{Hom}_{R}(-, C)$. Put $t=$ $\operatorname{depth}_{R} C$ and set $M=\operatorname{Tr} \Omega^{t} k$. Then we have $\operatorname{depth} R=t$ by [ $[$. Since

$$
\operatorname{grade}_{R} \operatorname{Ext}_{R}^{i}(k, R) \geq i-1
$$

for $1 \leq i \leq t$, the module $\Omega^{t} k$ is $t$-torsionfree by [1, Proposition (2.26)]. Hence $\operatorname{Ext}_{R}^{i}(M, R)=0$ for $1 \leq i \leq t$. As $M$ is free on the punctured spectrum of $R$, Lemma 2.3 implies $\operatorname{Ext}_{R}^{i}\left(M \otimes_{R} C, C\right)=0$ for $1 \leq i \leq t$. By assumption (3), the module $M \otimes_{R} C$ has an embedding into a module $X$ with $C$ - $\operatorname{dim}_{R} X<\infty$. According to [7, Lemma 4.3], we have $C-\operatorname{dim}_{R} X \leq t$. Lemma 2.2 shows that the natural map $\lambda_{M \otimes_{R} C}: M \otimes_{R} C \rightarrow\left(M \otimes_{R} C\right)^{\dagger \dagger}$ is injective. On the other hand, since there are natural isomorphisms

$$
\begin{aligned}
\left(M \otimes_{R} C\right)^{\dagger \dagger} & =\operatorname{Hom}_{R}\left(\operatorname{Hom}_{R}\left(M \otimes_{R} C, C\right), C\right) \cong \operatorname{Hom}_{R}\left(\operatorname{Hom}_{R}\left(M, \operatorname{Hom}_{R}(C, C)\right), C\right) \\
& \cong \operatorname{Hom}_{R}\left(\operatorname{Hom}_{R}(M, R), C\right),
\end{aligned}
$$

we see from [1, Proposition (2.6)(a)] that

$$
\begin{aligned}
\operatorname{Ker} \lambda_{M \otimes_{R} C} & \cong \operatorname{Ext}_{R}^{1}(\operatorname{Tr} M, C) \cong \operatorname{Ext}_{R}^{1}\left(\Omega^{t} k, C\right) \\
& \cong \operatorname{Ext}_{R}^{t+1}(k, C) .
\end{aligned}
$$


Thus we obtain $\operatorname{Ext}_{R}^{t+1}(k, C)=0$. By [3, Theorem (1.1)], the $R$-module $C$ must have finite injective dimension.

As we observed in the proof of the implication (1) $\Rightarrow(2)$, assertion (1) implies that $R$ is Cohen-Macaulay. Thus the last assertion follows.

Now we give applications of our main theorem. Letting $C=R$ in Theorem 1.3. we obtain the following result. This improves Theorem 1.2 and extends Theorem 1.1 .

Corollary 2.4. The following are equivalent:

(1) $R$ is Gorenstein.

(2) Every $R$-module can be embedded in an $R$-module of finite projective dimension.

Combining Corollary 2.4 with [4, Theorem 1], we have the following.

Corollary 2.5. If every finitely generated $R$-module can be embedded in a finitely generated $R$-module of finite projective dimension, then every finitely generated $R$-module can be embedded in a finitely generated $R$-module of finite injective dimension.

\section{ACKNOWLEDGMENTS}

The authors thank Sean Sather-Wagstaff and the referees for their kind comments and valuable suggestions.

\section{REFERENCES}

[1] M. Auslander; M. Bridger, Stable module theory. Memoirs of the American Mathematical Society, No. 94, American Mathematical Society, Providence, R.I., 1969. MR 0269685 $(42: 4580)$

[2] W. Bruns; J. Herzog, Cohen-Macaulay rings. Revised edition. Cambridge Studies in Advanced Mathematics, 39, Cambridge University Press, Cambridge, 1998. MR 1251956 (95h:13020)

[3] R. Fossum; H.-B. Foxby; P. Griffith; I. Reiten, Minimal injective resolutions with applications to dualizing modules and Gorenstein modules. Inst. Hautes Études Sci. Publ. Math. 45 (1975), 193-215. MR0396529(53:392)

[4] H.-B. Foxby, Embedding of modules over Gorenstein rings. Proc. Amer. Math. Soc. 36 (1972), 336-340. MR0309930 (46:9034)

[5] E. S. Golod, G-dimension and generalized perfect ideals (Russian). Algebraic geometry and its applications. Trudy Mat. Inst. Steklov. 165 (1984), 62-66. MR752933(85m:13011)

[6] R. Y. Sharp, Gorenstein modules. Math. Z. 115 (1970), 117-139. MR0263801 (41:8401)

[7] R. TAKAhashi, A new approximation theory which unifies spherical and Cohen-Macaulay approximations. J. Pure Appl. Algebra 208 (2007), no. 2, 617-634. MR2277700|(2007h:13016)

Department of Mathematical Sciences, Faculty of Science, Shinshu University, 3-1-1 Asahi, Matsumoto, Nagano 390-8621, Japan

E-mail address: takahasi@math.shinshu-u.ac.jp

Department of Mathematics, University of Tehran, P. O. Box 13145-448, Tehran, Iran - And - School of Mathematics, Institute for Research in Fundamental Sciences (IPM), P. O. Box 19395-5746, Tehran, Iran

E-mail address: yassemi@ipm.ir

Graduate School of Natural Science and Technology, Okayama University, Okayama 700-8530, JAPAN

E-mail address: yoshino@math.okayama-u.ac.jp 